\title{
Method Card Design Dimensions: A Survey of Card-Based Design Tools
}

\author{
Christiane Wölfel ${ }^{1,2}$ and Timothy Merritt ${ }^{3}$ \\ ${ }^{1}$ University of Munich, Amalienstraße 17, 80333 Munich, Germany \\ ${ }^{2}$ Aarhus University, Aabogade 34, 8200 Aarhus, Denmark \\ woelfel@cip.ifi.lmu.de \\ ${ }^{3}$ Aarhus School of Architecture, Nørreport 20, 8000 Aarhus, Denmark \\ Timothy.Merritt@aarch.dk
}

\begin{abstract}
There are many examples of cards used to assist or provide structure to the design process, yet there has not been a thorough articulation of the strengths and weaknesses of the various examples. We review eighteen cardbased design tools in order to understand how they might benefit designers. The card-based tools are explained in terms of five design dimensions including the intended purpose and scope of use, duration of use, methodology, customization, and formal/material qualities. Our analysis suggests three design patterns or archetypes for existing card-based design method tools and highlights unexplored areas in the design space. The paper concludes with recommendations for the future development of card-based methods for the field of interaction design.
\end{abstract}

Keywords: method cards, creativity cards, design methods, design tools.

\section{Introduction}

\section{'Design methods are like toothbrushes. \\ Everyone uses them, but no one likes to use someone else's.' [13]}

Physical cards have been popular design tools, perhaps because they are simple, tangible and easy to manipulate. Aside from the well-known Card Sorting method [22], cards have been used widely by designers to make the design process visible and less abstract [3,10] and serve as communication tools between members of the design team and users [9]. There are many examples of unique method card systems, many have similar features and formal qualities, yet it is not easy to get an overview of the available card systems in order to decide which to use, and when. As suggested in the opening quote attributed to John Zimmerman, designers often develop their own methods or appropriate widely known methods to best suit their needs, yet there is a tendency to use methods that are familiar instead of venturing out. Even though these methods are shared with the research community, it is difficult and time consuming for designers to review all available tools to understand their strengths and weaknesses. Therefore, this paper aims at providing an overview of some well-known sets of method cards including features and limitations so that the interaction designer can 
quickly review and make informed choices when selecting card-based method tools or to serve as a source of inspiration as they develop and appropriate a suite of design methods of their own.

We begin by analyzing different card systems according to five design dimensions, which suggest possible archetypes or patterns for these tools. We then discuss limitations and future work on card-based design tools that could benefit interaction design researchers.

\section{$2 \quad$ Analysis of Card-Based Design Tools}

There are many examples of cards being used to assist or provide structure to the design. We selected and examined eighteen card systems through direct use and reviewed the accompanying literature to gain an understanding of the authors' intentions for use. As first steps toward describing the various card-based systems, we identified five design dimensions. While this is not an exhaustive survey, it begins to provide a sketch of the card-based method landscape and highlights key differences among the attributes of many card-based tools. This approach has been used to articulate the design space of various examples of interactive media [20,26], and is used in the present paper to encourage the design community to engage and develop the field of card-based tools. When examined according to these dimensions, three broad groups of card systems emerge suggesting possible archetypes or design patterns [28]. A secondary contribution is that our review assists the review and selection of cards for design researchers and practitioners.

\subsection{Design Dimensions}

As stated previously, our aims in defining the five dimensions-and graduations within the dimensions - are in revealing key differences across the examples including: 1) Intended Purpose \& Scope, 2) Duration of use and placement in design process, 3) System or Methodology of use, 4) Customization, and 5) Formal Qualities. These attributes describe claims from the literature of the authors, the formal characteristics, and the tools in use. While these may seem closely related, it is an initial step in developing a framework for discussing the design attributes in card-based tools. Graduations within these dimensions were chosen to differentiate the examples-future work is needed, however to validate and develop these further.

Intended Purpose and Scope. Based on research literature or from booklets and inserts included in the card packages, the respective authors have made claims as to where their tools fit within the design process (ideation, inspiration, engaging nondesigners, etc.). In this category we can ask: where in the design process are the cards used and how should they be used? Do they have a specific purpose and do they focus on a particular context? We identified three graduations of intended purpose \& scope, ranging from very general to context specific. 
General/Repository card systems provide inspiration and challenge designers to take another point of view. An example here are the Oblique Cards [7, 8], which can be engaged with at any time in any context to increase lateral thinking and stimulate design problem solving in general. These types of cards aim for open-ended inspiration with little or no guidance on their use. These cards mainly function as repositories for design methods, capturing well-known methods from important literature [15] and offload the task of remembering the many design methods.

We also found various examples of cards, which focus on participatory design. They seek to develop sensitivity and empathy [24] for the context, and engage designers and users in the process. Some cards are designed for a better communication between users and designers, examples here are the Questionable Concept Cards [1], which encourage criticism and debate or the Inspiration Cards [2] that require collaborative work between designers and domain experts using the cards.

There are also context specific/ agenda-driven examples. This includes those cards focused on a particular context or design agenda as the Sound Design Deck [4], which facilitates sound design in games or the Design Play Cards [23], which focus on designing for sustainability.

Duration of Use/ When in Process. It is important to acknowledge the time investment that the various systems require - and to know when in the process they are used. This dimension includes key differences in the length of time ranging from one time use to sustained use of the system throughout the design process. Another aspect is the placement in the design process - whether the cards should be used in the very beginning, after initial field studies or prior to mockup sessions and prototyping. Four groups were identified, which range from anywhere/anytime to at a specific point in time.

The Oblique Cards are an example of cards that can be used anywhere/any time in the process. They can be useful in the very first phase of idea generation, but also when facing problems during the design, being stuck or looking for alternatives. Cards presenting a collection of methods as the IDEO Method Cards [16], are often positioned to be used as needed. As they provide a lot of different methods, some of those will fit in an early design stage, whereas others are for evaluation and testing. Other cards should be used at the beginning of the process as they provide input for further concept development; for example, PictureCARDs [12] are used after an initial field study and provide the basis for the card creation.

The last aspect of time is that cards are used at a specific point, for example in a workshop. The Sound Design Deck is used in this way, when applying the introduced methodology. But even though most of the work with the cards is done in a short session $(\sim 2 \mathrm{~h})$, one should still refer back to the cards later in the design process.

Methodology of Use. Some of the cards can be used very freely, whereas others provide a methodology how to use the cards. Some of the approaches are playful and game-like; some have rules or discreet steps that should be followed. This can be helpful to get started using the cards but might at the same time be restrictive. We 
identified three groups in this category: no methodology, suggestion for use and specific instructions.

Cards with no system are used ad-hoc with no suggested structured process provided by the authors. Cards of this type include IDEO [16], SUTD [14], and Oblique [7]. Most of the cards offer at least a basic suggestion for use. The DSKD Cards [17] come with a small brochure, which has some examples how the cards can be used. The authors of the PictureCARDs describe how they were using the cards, but there are no hard and fast, specific rules. The last category describes cards in which specific instructions are given. The authors of the Sound Design Cards introduced a specific method of how to use the cards, including a workspace with four regions in which cards can be moved, thus facilitating idea generation and keeping track of the design work at the same time. Inspiration Cards [2] also provide specific instructions, noting where the cards should be arranged on a poster to formulate a design idea.

Customization. Although we acknowledge that any technology tool will be adapted and appropriated into the user's life, in this dimension, we describe the degree to which the tool provides for customization as part of its use. The first group we identified in this category is no customization. When we examine the SUTD, Oblique, etc cards, they are intended to be static and unchanged. Cards offering trivial customization, do not allow the user to add or modify content, but only to structure or group the cards. This is the case with the IDEO iPhone app that in most respects replicates the paper cards [16] allowing the user to make groups and add cards to the groups. The Sound Design Deck provides for optional customization, whereas users can create their own cards and add them to a wiki. This is intended and welcome by the authors, as they aim to create a pattern language for sound design. The last group of cards requires customization in order to be utilized. Examples here are the Inspiration Cards, the Ideation Deck [6] or Questionable Concept Cards. The cards have to be created beforehand and are therefore applicable in the specific project, which helps the designer to get a better understanding of the project domain.

Formal Qualities. While the focus of this paper is on "cards", there are differences in the physical properties (2-sides, paper, size, shape), connections to virtual systems (stand alone or connected to objects in the room or in the virtual world), and appearances (images, diagrams, words, color schemes, etc.) Other formal qualities include issues such as the fact that some card systems have only one copy of a card vs. multiple copies, etc. We do not provide for all possible configurations, however, we provide graduations according to the use of media. The simplest type of cards have only text or only images, while most of the cards combine text and image or illustration, like the Inspiration Cards or the PLEX Cards [3]. The authors of PLEX Cards present their evaluation of the cards, and describe feedback regarding the images. This feedback highlights the importance of choosing suitable images for cards-they claim that the image should be abstract enough to allow an open interpretation, but at the same time detailed enough so that the user can relate to and interpret it. There are various card systems where the content is divided into different categories, as with the IDEO 
Method cards or the SUTD Cards, which provides thematic structure in the cards and suggests how the cards relate to each other. Finally, there are some cards, which have a virtual component, as in the Sound Design Deck, which connects the physical cards to the online wiki providing additional information and example videos.

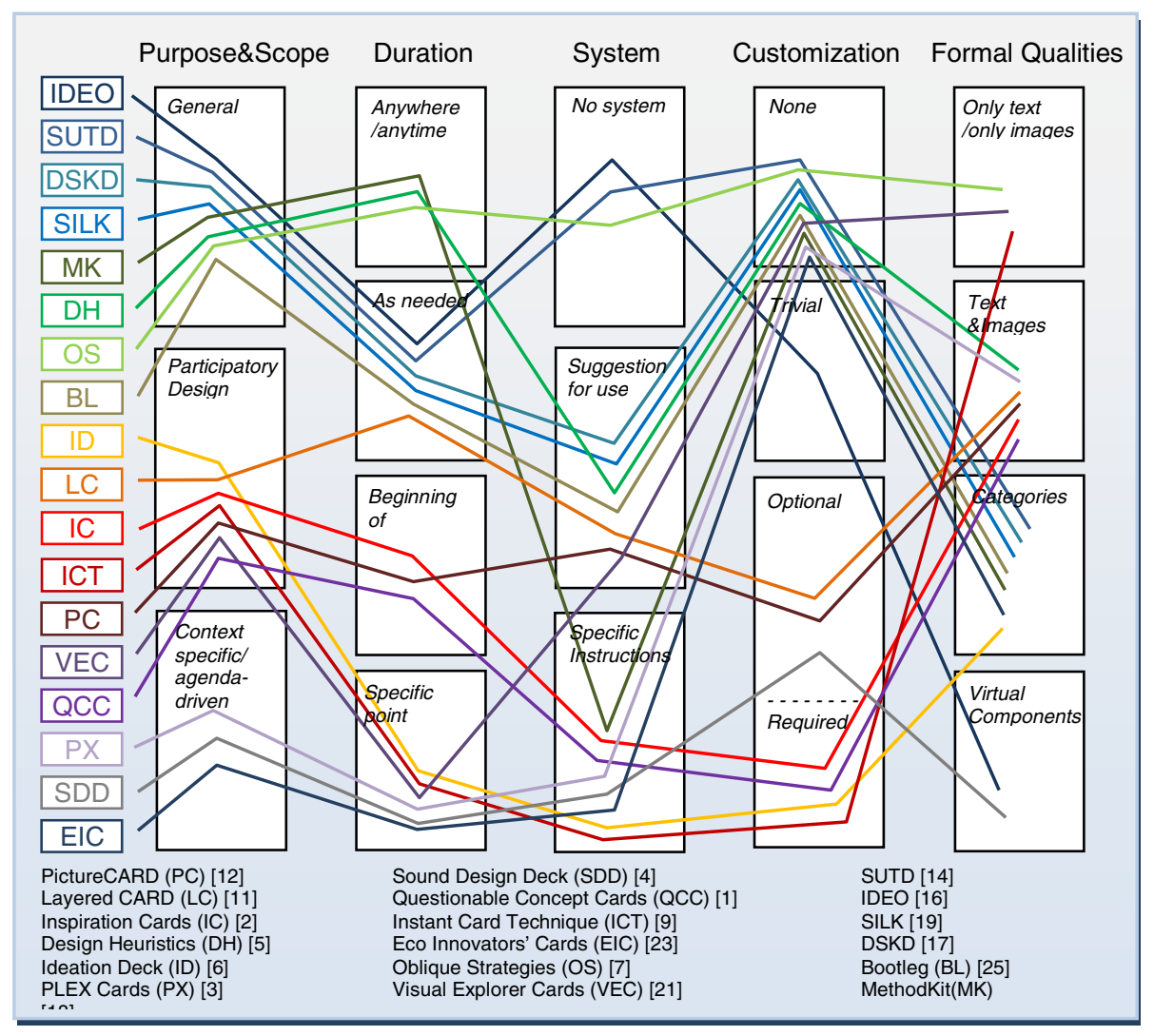

Fig. 1. Classification of method cards

\subsection{Toward Patterns of Design}

By plotting the card systems as shown in Figure 1, there are three archetypes or patterns of design [26,28] that emerge: general purpose/repository cards, customizable cards, and context specific cards. Further analysis and refinement of these groupings will be taken up in future work, however we can provide initial comments. Cards that are classified as "general purpose/repository" cards offer either a method repository or aim to stimulate inspiration and lateral thinking. They can be used during the whole process and only have general or no instruction for use. In most cases the deck is split up into different categories yet used without customization. "Customizable" cards are inherently customizable to some degree. Interestingly, we can see that most of these 
belong to the participatory design group and have specific instructions on how to use them to engage with end-users and non-designers. They are mainly used at a specific point in the design process and are composed of text and images. The third group includes "context specific" cards that are developed primarily to focus on a specific design agenda or context. They are primarily used at a specific point in time with specific instructions. In the next section we discuss how this exercise of analysis helps the design research community make sense of card-based tools.

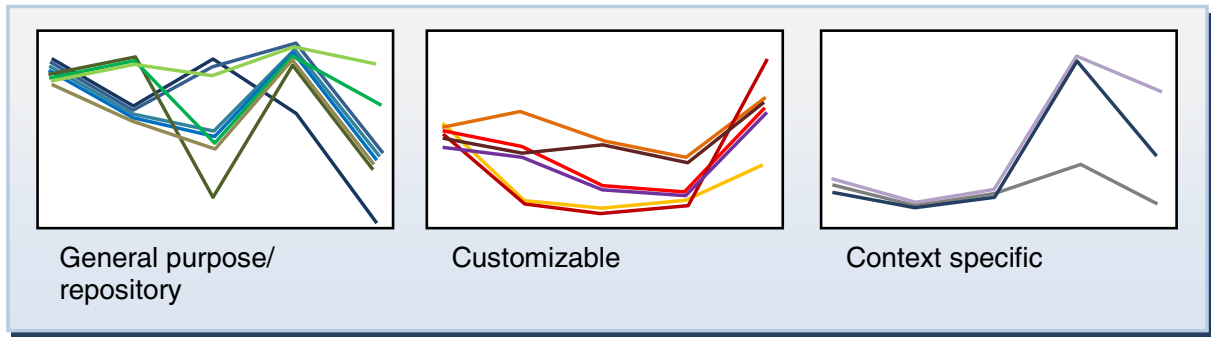

Fig. 2. Method card design patterns

\section{Discussion}

The classification exercise and resulting design patterns raise important issues for designers about when the cards can be used in the design process and what one should consider when choosing a card deck. We propose future work enabling customization and extending physical cards into digital media or augmented representations. To conclude, limitations of the present paper are provided.

\subsection{Using and Choosing Design Method Cards}

As noted earlier, designers can choose to use method cards in their design process either by using existing systems or by creating a new set of cards—essentially, should the designer use an existing toothbrush [13] or create a new one? This decision depends on several factors including time, knowledge of team members, needs of the design team, expected results and many other possible factors. Through the process of reviewing and analyzing the card systems and accompanying literature it would seem beneficial for designers to become familiar with at least one example of each of the three card archetypes we identified before choosing or creating a card-based tool. After reviewing these, the designer should evaluate the time and resource investment that can be made and to stimulate ideas about possible formats for a card system if they determine that they will build a tool to be used on an ongoing basis. If a new card system is created, perhaps the most critical attribute is the degree of customization that is expected-whether the cards are living and evolving, or serve more as a lasting resource of commonly needed methods. In the next section we engage with the issue of customization of card systems and the use of technology as ways to extend traditional card-based tools. 


\subsection{Customization and Use of New Technology}

It was surprising that only few examples were found that offer customization directly, or in some form of digital component to augment the tool. There is much interest in the development of robust technologies including QR codes, augmented reality, e-ink, etc. that bridge the gap between the digital and physical world with the aim of engaging people into complicated work processes [27] or to capture lasting impressions of work. Showing similarities between individual cards within different card systems could go much further to reduce the time spent in the design process searching for appropriate methods. Various digital technologies could facilitate this by showing connections virtually or presenting additional information augmented onto the cards on the table or wall. Designers could add their own notes to cards and share those with their team members, which was a future development suggested also in [2], yet robust examples were not found in any of the card system except in [4], however, it is not clear that the digital components are used for anything more than a repository. This does however go beyond the digital representation approach offered in the iPhone App for the IDEO Method Cards. There is no connection to the physical world except that all of the digital cards are copies of the physical cards-there is no customization other than adding cards to groups. In a sense, the digital app loses functionality - the designer can not look at more than one card at a time, can not write on the card itself, and there is no new information despite the content being more than ten years old. In our future work we intend to explore meaningful ways of connecting physical card-based tools with interactive digital elements.

\subsection{Limitations}

While the present paper begins to sketch out the design space for design method cards, there are limitations that we would like to acknowledge. Our list of method cards does not represent all method card systems, yet eighteen examples is adequate to begin to understand the design space. We also did not provide in-depth reviews of each of the card systems, excluded the number of cards in each deck, etc. These limitations do not diminish the contribution here, but rather signal important future work.

\section{References}

1. Vines, J., Blythe, M., Lindsay, S., Dunphy, P., Monk, A., Olivier, P.: Questionable concepts: critique as resource for designing with eighty somethings. In: Proc. CHI 2012, pp. 1169-1178. ACM, NY (2012)

2. Halskov, K., Dalsgaard, P.: Inspiration card workshops. In: Proc. DIS 2006, pp. 2-11. ACM, NY (2006)

3. Lucero, A., Arrasvuori, J.: PLEX cards: a source of inspiration when designing for playfulness. In: Proc. Fun and Games 2010, pp. 28-37. ACM, NY (2010)

4. Alves, V., Roque, L.: A deck for sound design in games: enhancements based on a design exercise. In: Proc. ACE 2011. ACM, NY (2011) 
5. Daly, S.R., et al.: Assessing Design Heuristics for Idea Generation in an Introductory Engineering Course. International Journal of Engineering Education 28(2), 463 (2012)

6. Golembewski, M., Selby, M.: Ideation decks: a card-based design ideation tool. In: Proc. DIS 2010, pp. 89-92. ACM, NY (2010)

7. Eno, B., Schmidt, P.: Oblique strategies. Opal, London (1978)

8. Burns, A.: Oblique Strategies for Ambient Journalism. M/C Journal (May 2010), http: / / www. journal. mediaculture.org.au/index.php/mcjournal/article/view/230

9. Beck, E., Obrist, M., Bernhaupt, R., Tscheligi, M.: Instant card technique: how and why to apply in user-centered design. In: Proc. PDC 2008, pp. 162-165. ACM, NY (2008)

10. Lafrenière, D., Dayton, T., Muller, M.: Variations of a theme: card-based techniques for participatory analysis and design. In: Proc. CHI 1999, pp. 151-152. ACM, NY (1999)

11. Muller, M.J.: Layered participatory analysis: new developments in the CARD technique. In: Proc. CHI 2001, pp. 90-97. ACM, NY (2001)

12. Tschudy, M., Dykstra-Erickson, E., Holloway, M.: PictureCARD: A Storytelling Tool for Task Analysis. In: Proc. PDC 1996, Cambridge, MA, USA, November 13-15 (1996)

13. Harrison, S., Tatar, D.: On Methods. Interactions 18(2), 10-11 (2011)

14. Singapore University of Technology Design: SUTD Method Cards (2012), http: / / design. sutd.edu.sg/wpcontent/uploads / files / extraResources/MethodCards.pdf

15. Rogers, Y., Sharp, H., Preece, J.: Interaction Design: Beyond Human - Computer Interaction, 3rd edn. Wiley (June 2011)

16. IDEO: IDEO Method Cards: 51 Ways to Inspire Design. IDEO, Palo Alto (2003)

17. Kolding School of Design: DSKD Method Cards (2011), http: / / www. designskolenkolding.dk/index . php?id=2241

18. Möller, O.: MethodKit. Idea Society, Stockholm, Sweden (2013)

19. SILK (Social Innovation Lab Kent): Method Deck. Kent, UK (2007)

20. Cheok, A.D., Kok, R.T., Tan, C., Fernando, O.N.N., Merritt, T., Sen, J.Y.P.: Empathetic living media. In: Proc. DIS 2008, pp. 465-473. ACM, NY (2008)

21. Center for Creative Leadership: Visual Explorer Post Card Facilitator's Set. Greensboro, NC (2013),

http://solutions.ccl.org/Visual_Explorer_Post_Card_Facilitat or's_set

22. Hudson, W.: Playing your cards right: getting the most from card sorting for navigation design. Interactions 12(5), 56-58 (2005)

23. Eco Innovators: Design Play Cards: Designing for Sustainability. Melbourne (2013)

24. Bowen, G.: Grounded theory and sensitizing concepts. Int. J. Qual. Meth. 5(3) (2006)

25. Stanford University Institute of Design: Bootcamp Bootleg (2010), http: //dschool. stanford. edu/wpcontent/uploads/2011/03/BootcampBootleg2010v2SLIM.pdf

26. Pousman, Z., Stasko, J.: A taxonomy of ambient information systems: four patterns of design. In: Proc. AVI 2006, pp. 67-74. ACM, New York (2006)

27. Zhao, S., Nakamura, K., Ishii, K., Igarashi, T.: Magic cards: a paper tag interface for implicit robot control. In: Proc. CHI 2009, pp. 173-182. ACM, NY (2009)

28. Alexander, C.: A Pattern Language: Towns, Buildings, Construction. Oxford University Press, UK (1977) 